Research Article

\title{
Periodic Points of Asymmetric Bernoulli Shifts
}

\author{
Yong-Guo Shi $\mathbb{D},{ }^{1}$ Kai Chen $\mathbb{D}^{2},{ }^{2}$ and Wei Liao ${ }^{1}$ \\ ${ }^{1}$ College of Mathematics and Information Science, Neijiang Normal University, Neijiang 641100, Sichuan, China \\ ${ }^{2}$ Department of Mathematics, Sichuan University, Chengdu 610064, Sichuan, China
}

Correspondence should be addressed to Kai Chen; 13709047269@163.com

Received 1 September 2021; Accepted 16 September 2021; Published 14 October 2021

Academic Editor: Wenpeng Zhang

Copyright $(2021$ Yong-Guo Shi et al. This is an open access article distributed under the Creative Commons Attribution License, which permits unrestricted use, distribution, and reproduction in any medium, provided the original work is properly cited.

It is well-known that Sharkovskii's theorem gives a complete structure of periodic order for a continuous self-map on a closed bounded interval. As a further study, a natural problem is how to determine the location and number of periodic points for a specific map. This paper considers the periodic points of asymmetric Bernoulli shift, which is a piecewise linear chaotic map.

\section{Introduction}

In 1964, Sharkovskii [1] firstly introduced a special ordering on the set of positive integers. This ordering implies that if $p \triangleleft q$ and a continuous self-map of a closed bounded interval has a point of period $p$; then it has a point of period $q$. The least number with respect to this ordering is 3 . Thus, if a map has a point of period 3 , then it has points of any periods. In 1975, the latter result was rediscovered by Li and Yorke [2]. Then numerous papers are devoted to the study of interval maps (see e.g., [3-5] and references therein).

Bifurcation points of some interval maps were studied in [6], and the limit behavior of orbits and probabilistic some problems were considered in $[7,8]$. Recently, Ivanov in [9] considered an exact lower bound for the number of orbits of a given period for a self-map of a closed bounded interval.

Consider the asymmetric Bernoulli shift $F:[0,1] \longrightarrow$ $[0,1]$ with a parameter $0<a<1$, defined by

$$
F(x)= \begin{cases}\frac{x}{a}, & 0 \leq x \leq a, \\ \frac{x-a}{1-a}, & a<x \leq 1 .\end{cases}
$$

Specially, when $a=1 / 2$, it is the Bernoulli Shift or the binary transformation, also known as doubling map or the binary transformation. Conjugacies between asymmetric Bernoulli shifts are constructed in [10].
Given a positive integer $n$, one interesting question is how to find all $n$-periodic points of $F$. The other is how many $n$-periodic points of $F$.

In this paper, we study periodic orbits of $F$. In the next section, we present dynamics of jumps of $F^{n}$. Section 3 recalls the real number representation, i.e, $F$-expansion. In Section 4, we use the $F$-expansion to give explicit formulas of $F^{n}(x)$ for $n \in \mathbb{N}$, explicit formulas of jumps of $F^{n}(x)$, explicit formulae of fixed points of $F^{n}(x)$, and explicit formulas of all $n$-periodic points of $F(x)$. The last section gives the number $h(n)$ of periodic orbits of a given period $n$ for $F$ and the limit behavior of $h(n)$.

\section{Dynamics of Jumps of $F^{n}$}

For $n \in \mathbb{N}$, let $F^{n}(x)$ denote the $n$-th iterate of $F$, which is recursively defined by $F^{0}(x)=x$ and $F^{n}=F\left(F^{n-1}(x)\right)$ for $x \in[0,1]$.

A point $c \in(0,1)$ is called a jump of $F$ if the one-sided limits, $F(c-)$ and $F(c+)$, exist and are finite, but are not equal. The set of jumps of $F$ is denoted by $\mathscr{J}(F)$. One can see that

$$
\mathscr{J}(F) \subseteq \mathscr{J}\left(F^{2}\right) \subseteq \cdots \subseteq \mathscr{J}\left(F^{n}\right) \subseteq \mathscr{J}\left(F^{n+1}\right) \subseteq \cdots
$$

Each element of $\mathscr{J}\left(F^{n+1}\right) \backslash \mathscr{J}\left(F^{n}\right)$ must be a preimage under $F$ of a point from $\mathscr{J}\left(F^{n}\right)$. More precisely, 


$$
\mathscr{J}\left(F^{n+1}\right) \backslash \mathscr{J}\left(F^{n}\right)=F^{-1}\left(\mathscr{J}\left(F^{n}\right)\right) \backslash \mathscr{J}\left(F^{n}\right) .
$$

The map $F$ has the unique jump $a$. Put $x_{1,0}:=0, x_{1,1}:=a$, and $x_{1,2}:=1$. Let $I$ denote the unit interval $[0,1]$, $I_{1,1}:=\left(x_{1,0}, x_{1,1}\right)$, and $I_{1,2}:=\left(x_{1,1}, x_{1,2}\right)$. One can see that $F^{n}$ has $2^{n}-1$ jumps for $n \geq 2$ by induction. For $i, j \in \mathbb{N}^{+}$, let $x_{i, 0}:=0, x_{i, 2^{i}}:=1$, and $x_{i, j}$ denote the $j$ th jumps of $F^{i}$ in the following order:

$$
0=x_{i, 0}<x_{i, 1}<x_{i, 2}<\cdots<x_{i, j}<\cdots<x_{i, 2^{i}-1}<x_{i, 2^{i}}=1 .
$$

Put $I_{i, j}:=\left(x_{i, j-1}, x_{i, j}\right)$ for every $j \in\left\{1,2,3, \ldots, 2^{i}\right\}$. It is clear that $I_{i, j}$ is the $j$-th monotonic interval of $F^{i}$.

Lemma 1. For $n \geq 1$, the jumps of $F^{n}$ and $F^{n-1}$ have the following relationship:

$$
\begin{aligned}
& \text { (i) } F\left(x_{n, k}\right)=F\left(x_{n, 2^{n-1}+k}\right)=x_{n-1, k} \text { for } 1 \leq k \leq 2^{n-1}-1 \\
& \text { (ii) } x_{n, 2^{n-1}}=x_{n-1,2^{n-2}}=a \\
& \text { (iii) } F^{i}\left(I_{n, k}\right)=F^{i}\left(I_{n, 2^{n-1}+k}\right)=I_{n-i, k} \text { for } 1 \leq k \leq 2^{n-i} \text { and } \\
& 1 \leq i \leq n-1
\end{aligned}
$$

Proof. We first claim that $a$ is a jump of $F^{n}$ for every $n \geq 1$. In fact, since $a$ is a jump of $F(x), a$ is also a jump of $F^{n}(x)$ for $n \geq 2$. Moreover, it is easy to check that $F^{n}(a)=1$ for $n \geq 2$.

Next, we prove (i) and (ii) by induction. It is clear that these results holds for $n=2$.

Assume that these results hold for $n=m \geq 2$, i.e.,

(i) $F\left(x_{m, k}\right)=F\left(x_{m, 2^{m-1}+k}\right)=x_{m-1, k}$ for $1 \leq k \leq 2^{m-1}-1$

(ii) $x_{m, 2^{m-1}}=x_{m-1,2^{m-2}}=a$

Now we shall prove these results hold for $n=m+1$. Denote $2^{m}-1$ jumps of $F^{m}$ by

$$
0<x_{m, 1}<x_{m, 2}<\cdots<x_{m, k}<\cdots<x_{m, 2^{m}-1}<1 \text {. }
$$

Since $F$ is strictly increasing on the subinterval $I_{1,1}$ and $F\left(I_{1,1}\right)=(0,1)$, for each $k \in\left\{1,2, \ldots, 2^{m}-1\right\}$, there exists the unique point, denoted by $x_{m+1, k}$, in $I_{1,1}$ such that $F\left(x_{m+1, k}\right)=x_{m, k}$. Since $F$ is strictly increasing on $I_{1,1}$, one can see that

$$
0<x_{m+1,1}<x_{m+1,2}<\cdots<x_{m+1, k}<\cdots<x_{m+1,2^{m}-1}<a .
$$

Further, by the definition of jump, $x_{m+1, k}$ is a jump of $F^{m+1}=F^{m} \circ F$ for each $k \in\left\{1,2, \ldots, 2^{m}-1\right\}$.

Similarly, since $F$ is strictly increasing on the subinterval $I_{1,2}$ and $F\left(I_{1,2}\right)=(0,1)$, for each $k \in\left\{1,2, \ldots, 2^{m}-1\right\}$, there exists the unique point, denoted by $x_{m+1,2^{m}+k}$, in $I_{1,2}$ such that $F\left(x_{m+1,2^{m}+k}\right)=x_{m, k}$. Since $F$ is strictly increasing on $I_{1,2}$, one can see that

$$
\begin{aligned}
a & <x_{m+1,2^{m}+1}<x_{m+1,2^{m}+2}<\cdots<x_{m+1,2^{m}+k} \\
& <\cdots<x_{m+1,2^{m+1}-1}<1 .
\end{aligned}
$$

Further, by the definition of jump, $x_{m+1,2^{m}+k}$ is a jump of $F^{m+1}=F^{m} \circ F$ for each $k \in\left\{1,2, \ldots, 2^{m}-1\right\}$. Let $x_{m+1,2^{m}}$ denote $a$. Therefore,

(i) $F\left(x_{m+1, k}\right)=F\left(x_{m+1,2^{m}+k}\right)=x_{m, k}$ for $1 \leq k \leq 2^{m}-1$

(ii) $x_{m+1,2^{m}}=x_{m, 2^{m-1}}=a$

It follows from (i) that for $1 \leq k \leq 2^{n-1}$,

$$
F\left(I_{n, k}\right)=F\left(I_{n, 2^{n-1}+k}\right)=I_{n-1, k} .
$$

Then for $1 \leq k \leq 2^{n-i}$ and $1 \leq i \leq n-1$,

$$
F^{i}\left(I_{n, k}\right)=F^{i}\left(I_{n, 2^{n-1}+k}\right)=I_{n-i, k} .
$$

This completes the proof.

\section{F-Expansion}

In this section, we will introduce a new real number representation.

Definition 1. A sequence $\left\{\varepsilon_{k}\right\}_{k \in \mathbb{N}^{+}}$of 0 and 1 is called the itinerary of $x \in[0,1]$ with respect to the asymmetric Bernoulli shift $F:[0,1] \longrightarrow[0,1]$ and $a \in(0,1)$, if, for $k \geq 1$,

$$
\varepsilon_{k}= \begin{cases}0, & F^{k-1}(x) \leq a, \\ 1, & F^{k-1}(x)>a .\end{cases}
$$

In fact, the itinerary of $x \in[0,1]$ with respect to $F$ and $a \in(0,1)$ is just the $F$-expansion of a real $x \in[0,1]$. According to [10], or these two classic papers [11, 12], we have an expansion for $x$ in powers of the numbers $a$ and $1-a$ :

$$
x=\sum_{k=1}^{\infty} \varepsilon_{k} a^{k-s_{k-1}}(1-a)^{s_{k-1}}=\sum_{k=1}^{\infty} \varepsilon_{k} a^{k}\left(\frac{1-a}{a}\right)^{s_{k-1}},
$$

where $s_{0}=0$ and $s_{k}:=\sum_{j=1}^{k} \varepsilon_{j}$ for $k \geq 1$.

Thus, every $x \in[0,1]$ can be represented through its digit sequence $\left\{\varepsilon_{k}\right\}_{k \in \mathbb{N}^{+}}$. In this situation, write $x=\left[\varepsilon_{1}, \varepsilon_{2}, \ldots, \varepsilon_{k}, \ldots\right]$ for short. One can see that every infinite $F$-expansion is unique, whereas each $x \in(0,1)$ with a finite $F$-expansion can be expanded in exactly two ways, namely, one immediately verifies that

$$
x=\left[\varepsilon_{1}, \ldots, \varepsilon_{k-1}, 1\right]=\left[\varepsilon_{1}, \ldots, \varepsilon_{k-1}, 0,1,1,1, \ldots\right] .
$$

In the following, we employ a convention in which finite fractions such as

$$
\begin{aligned}
& {[1,1,0,0,0,0, \ldots]=[1,0,1,1,1,1, \ldots],} \\
& {[1,0,1,0,0,0, \ldots]=[1,0,0,1,1,1, \ldots],}
\end{aligned}
$$

are represented as finite fractions with infinite zeros, as $[1,1,0,0,0, \ldots]$ or $[1,0,1,0,0,0, \ldots]$, unless otherwise stated. 
Lemma 2. If $x=\left[\varepsilon_{1}, \varepsilon_{2}, \ldots, \varepsilon_{k}, \ldots\right]$, then $F(x)=\left[\varepsilon_{2}, \varepsilon_{3}\right.$, $\left.\ldots, \varepsilon_{k}, \ldots\right]$.

Proof. It follows from a property of the asymmetric Bernoulli shift $F(x)$ that $x \leq a$ provided that $\varepsilon_{1}=0$ in $x=\left[\varepsilon_{1}, \varepsilon_{2}, \ldots, \varepsilon_{k}, \ldots\right]$. One then finds

$$
\begin{aligned}
F(x) & =\frac{x}{a}=\sum_{k=1}^{\infty} \varepsilon_{k} a^{k-1}\left(\frac{1-a}{a}\right)^{s_{k-1}} \\
& =\sum_{k=2}^{\infty} \varepsilon_{k} a^{k-1}\left(\frac{1-a}{a}\right)^{s_{k-1}} \\
& =\sum_{k=1}^{\infty} \varepsilon_{k+1} a^{k}\left(\frac{1-a}{a}\right)^{s_{k}} \\
& =\left[\varepsilon_{2}, \varepsilon_{3}, \ldots, \varepsilon_{k}, \ldots\right] .
\end{aligned}
$$

On the other hand, one has $a<x \leq 1$ if $\varepsilon_{1}=1$ and hence

$$
\begin{aligned}
F(x) & =\frac{x-a}{1-a}=\sum_{k=2}^{\infty} \varepsilon_{k} a^{k-s_{k-1}}(1-a)^{s_{k-1}-1} \\
& =\sum_{k=1}^{\infty} \varepsilon_{k+1} a^{k+1-s_{k}}(1-a)^{s_{k}-1} \\
& =\left[\varepsilon_{2}, \varepsilon_{3}, \ldots, \varepsilon_{k}, \ldots\right] .
\end{aligned}
$$

This shows that, from the perspective of symbolic dynamics, $F$ corresponds to the shift map on the space $\{0,1\}^{\mathbb{N}^{+}}$, at least for those points with an infinite $F$-expansion.

One easily finds that the periodicity of the orbits is related to recurring $F$-expansions. For example,

$$
[1,0,1,1,1,1,0,1,1,1, \ldots] \text {, }
$$

is a recurring $F$-expansion with the recurring unit of the length 5 , and hence, it is a 5-periodic point of $F$.

\section{The Explicit Formula of $F^{n}$}

Since $F^{n}$ is a piecewise linear map, and $F^{n}$ is strictly increasing on each subinterval $I_{n, k}$. One can obtain the explicit formula of $F^{n}$.

Theorem 1. If $x=\left[\varepsilon_{1}, \varepsilon_{2}, \ldots, \varepsilon_{k}, \ldots\right]$, then

$$
\begin{aligned}
F^{n}(x)= & \frac{x}{a^{n-s_{n}}(1-a)^{s_{n}}} \\
& -\sum_{j=1}^{n} \frac{\varepsilon_{j}}{a^{n-j-s_{n}+s_{j}-1}(1-a)^{s_{n}-s_{j}+1}}, \quad \text { for } n \in \mathbb{N}^{+} .
\end{aligned}
$$

Proof. We prove this result by mathematical induction.

We firstly consider the trivial case $n=1$. If $x \in I_{1,1}$, then $\varepsilon_{1}=0$ and $s_{1}=0$. Thus,

$$
\begin{aligned}
F(x)= & \frac{x}{a^{1-s_{1}}(1-a)^{s_{1}}} \\
& -\sum_{j=1}^{1} \frac{\varepsilon_{j}}{a^{1-j-s_{1}+s_{j}-1}(1-a)^{s_{1}-s_{j}+1}}=\frac{x}{a} .
\end{aligned}
$$

If $x \in I_{1,2}$, then $\varepsilon_{1}=1$ and $s_{1}=1$. Thus,

$$
\begin{aligned}
F(x) & =\frac{x}{a^{1-s_{1}}(1-a)^{s_{1}}}-\sum_{j=1}^{1} \frac{\varepsilon_{j}}{a^{1-j-s_{1}+s_{j}-1}(1-a)^{s_{1}-s_{j}+1}} \\
& =\frac{x}{1-a}-\frac{a}{1-a} .
\end{aligned}
$$

Therefore, the result holds for $n=1$.

Assume that the result holds for $n=m \geq 1$, i.e.,

$$
F^{m}(x)=\frac{x}{a^{m-s_{m}}(1-a)^{s_{m}}}-\sum_{j=1}^{m} \frac{\varepsilon_{j}}{a^{m-j-s_{m}+s_{j}-1}(1-a)^{s_{m}-s_{j}+1}} .
$$

Now we shall prove that the result holds for $n=m+1$. If $F^{m}(x) \leq a$; then $\varepsilon_{m+1}=0$ and $s_{m+1}=s_{m}$. Thus,

$$
\begin{aligned}
F^{m+1}(x) & =\frac{1}{a} \cdot\left(\frac{x}{a^{m-s_{m}}(1-a)^{s_{m}}}-\sum_{j=1}^{m} \frac{\varepsilon_{j}}{a^{m-j-s_{m}+s_{j}-1}(1-a)^{s_{m}-s_{j}+1}}\right) \\
& =\frac{x}{a^{m+1-s_{m+1}}(1-a)^{s_{m+1}}}-\sum_{j=1}^{m+1} \frac{\varepsilon_{j}}{a^{m+1-j-s_{m+1}+s_{j}-1}(1-a)^{s_{m+1}-s_{j}+1}} .
\end{aligned}
$$

If $F^{m}(x)>a$; then $\varepsilon_{m+1}=1$ and $s_{m+1}=s_{m}+1$. Thus, 


$$
\begin{aligned}
F^{m+1}(x) & =\frac{1}{1-a} \cdot\left(\frac{x}{a^{m-s_{m}}(1-a)^{s_{m}}}-\sum_{j=1}^{m} \frac{\varepsilon_{j}}{a^{m-j-s_{m}+s_{j}-1}(1-a)^{s_{m}-s_{j}+1}}\right)-\frac{a}{1-a} \\
& =\frac{x}{a^{m-s_{m}}(1-a)^{s_{m}+1}}-\sum_{j=1}^{m} \frac{\varepsilon_{j}}{a^{m-j-s_{m}+s_{j}-1}(1-a)^{s_{m}-s_{j}+1+1}}-\frac{a}{1-a} \\
& =\frac{x}{a^{m+1-s_{m+1}}(1-a)^{s_{m+1}}}-\sum_{j=1}^{m} \frac{\varepsilon_{j}}{a^{m+1-j-s_{m+1}+s_{j}-1}(1-a)^{s_{m+1}-s_{j}+1}}-\frac{a}{1-a} \\
& =\frac{x}{a^{m+1-s_{m+1}}(1-a)^{s_{m+1}}}-\sum_{j=1}^{m+1} \frac{\varepsilon_{j}}{a^{m+1-j-s_{m+1}+s_{j}-1}(1-a)^{s_{m+1}-s_{j}+1}} .
\end{aligned}
$$

Therefore, the result holds for $n=m+1$. The proof is completed.

As a corollary, we present the exact formulas of these jumps of $F^{n}$.

Corollary 1. All jumps of $F^{n}$ are given by

$$
\sum_{j=1}^{n} \varepsilon_{j} a^{j+1-s_{j}}(1-a)^{s_{j}-1},
$$

where $\varepsilon_{j}=0$ or 1 , and not all are $\varepsilon_{j}$ equal to 0 .

Proof. If all $\varepsilon_{j}$ are zero, then $x_{1,0}=0$, and it is not a jump. From Theorem 2, solving $F^{n}(x)=0$, we can obtain all these jumps of $F^{n}$.

Definition 2. A point $x$ in $X$ is called a periodic point of a self-mapping $f: X \longrightarrow X$ if there exists an positive integer $n$ such that

$$
f^{n}(x)=x .
$$

The smallest positive integer $n$ satisfying the above is called the prime period or least period of the point $x$, the point $x$ is called an $n$-periodic point of $f$, and the sequence $\left\{x, f(x), \ldots, f^{n-1}(x)\right\}$ is called an $n$-periodic orbit.

In particularly, an 1-periodic point is called a fixed point.

The following corollary presents the exact formulas of all fixed points of $F^{n}$.

Corollary 2. All fixed points of $F^{n}$ are given by

$$
\frac{1}{1-a^{n-s_{n}}(1-a)^{s_{n}}} \sum_{j=1}^{n} \varepsilon_{j} a^{j+1-s_{j}}(1-a)^{s_{j}-1},
$$

where $\varepsilon_{j}=0$ or 1 .

Proof. Since the curve of $y=F^{n}(x)$ intersects the line of $y=x$ at $2^{n}$ points, $F^{n}(x)$ has $2^{n}$ fixed points. Solving $F^{n}(x)=x$, we have

$$
x=\frac{1}{1-a^{n-s_{n}}(1-a)^{s_{n}}} \sum_{j=1}^{n} \varepsilon_{j} a^{j+1-s_{j}}(1-a)^{s_{j}-1} .
$$

\section{The Number of $n$-Periodic Points}

The fixed points of $F$ are the intersections of $y=F(x)$ and $y=x$, namely, two points $x=0,1$. The intersections of $y=F^{2}(x)$ and $y=x$ have four points where there are two 2 -periodic points, namely, two points

$$
\begin{aligned}
& \frac{a^{2}}{1-a+a^{2}}, \\
& \frac{a}{1-a+a^{2}} .
\end{aligned}
$$

The other two intersections $x=0$ and 1 are the fixed points. The intersections of $y=F^{3}(x)$ and $y=x$ have eight points where there are six 3-periodic points and two fixed points.

In general, the intersections of $y=F^{n}(x)$ and $y=x$ have $2^{n}$ periodic points. If $x$ is a $p$-periodic point of $F$, then $p \mid n$. Let $h(p)$ denote the number of the $p$-periodic points. Then,

$$
\sum_{p \mid n} h(p)=2^{n}, \quad \text { for every integer } n \geq 1 \text {, }
$$

where the sum extends over all positive divisors $p$ of $n$.

In order to obtain the exact number $h(n)$ of $n$-periodic points of $F$, we need to introduce the Möbius function and Möbius inversion formula (see, for example, $[13,14]$ ).

Define the Möblius function $\mu: \mathbb{N} \longrightarrow\{1,0,1\}$ by

$$
\mu(n)= \begin{cases}1, & n=1, \\ (-1)^{r}, & n=q_{1}, q_{2}, \ldots, q_{r}, q_{1}<q_{2}<\cdots<q_{r}, \\ 0, & \text { others. }\end{cases}
$$

Thus, if $\left(n_{1}, n_{2}\right)=1$, then $\mu\left(n_{1} n_{2}\right)=\mu\left(n_{1}\right) \mu\left(n_{2}\right)$, and for any $n \in \mathbb{N}$, there holds

$$
\sum_{k \mid n} \mu(k)=\left\lfloor\frac{1}{n}\right\rfloor
$$

Lemma 3 (Möbius inversion formula). If $h$ and $g$ are arithmetic functions, i.e., from $\mathbb{N}$ to $\mathbb{C}$, satisfying 


$$
g(n)=\sum_{d \mid n} h(d), \quad \text { for every integer } n \geq 1
$$

Then,

$$
h(n)=\sum_{d \mid n} \mu(d) g\left(\frac{n}{d}\right), \quad \text { for every integer } n \geq 1,
$$

where $\mu$ is the Möbius function and the sums extend over all positive divisors $d$ of $n$.

In effect, the original $h(n)$ can be determined given $g(n)$ by using the inversion formula.

Corollary 3. The number of n-periodic points of $F$ is given by,

$$
h(n)=\sum_{d \mid n} \mu\left(\frac{n}{d}\right) 2^{d}, \quad \text { for every integer } n \geq 1 .
$$

Let $N(n)$ denote the number of $n$-periodic orbits of $F$. Then,

$$
N(n)=\frac{h(n)}{n}=\frac{1}{n} \sum_{d \mid n} \mu\left(\frac{n}{d}\right) 2^{d}, \quad \text { for every integer } n \geq 1 .
$$

The first several $N(n)$ are

$$
\begin{aligned}
& N(1)=2, \\
& N(2)=1, \\
& N(3)=2, \\
& N(4)=3, \\
& N(5)=6, \\
& N(6)=9, \\
& N(7)=18, \\
& N(8)=30,
\end{aligned}
$$

while $N(n)$ for larger $n$ are

$$
\begin{aligned}
& N(16)=4080 \\
& N(20)=52377 \\
& N(32)=134215680 \\
& N(64)=288230376084602880
\end{aligned}
$$

If the values just above are compared to

$$
\begin{aligned}
& \frac{2^{16}}{16}=4096 \\
& \frac{2^{20}}{20}=52428.8 \\
& \frac{2^{32}}{32}=134217728 \\
& \frac{2^{64}}{64}=288230376151711744
\end{aligned}
$$

one finds that the ratio of $N(n)$ and $\left(2^{n} / n\right)$ approaches 1 as $n \longrightarrow+\infty$.

Now we shall prove that the ratio of $h(n)$ and $2^{n}$ approaches 1 as $n \longrightarrow+\infty$.

Theorem 2. Let $h(p)$ be the number of the p-periodic points of F. Then,

$$
\lim _{n \longrightarrow+\infty} \frac{h(n)}{2^{n}}=\lim _{n \longrightarrow+\infty} \frac{\sum_{d \mid n} \mu(n / d) 2^{d}}{2^{n}}=1,
$$

where $\mu$ is the Möbius function.

Proof. On one hand,

$$
\begin{aligned}
\sum_{d \mid n} \mu\left(\frac{n}{d}\right) 2^{d} & \leq \sum_{d \mid n} 2^{d} \\
& =2^{n}+\sum_{d \mid n, d \neq n} 2^{d} \\
& \leq 2^{n}+\sum_{1 \leq d \leq\lfloor n / 2\rfloor} 2^{d} \\
& \leq 2^{n}+2^{(n / 2)+1}-2 .
\end{aligned}
$$

On the other hand,

$$
\begin{aligned}
\sum_{d \mid n} \mu\left(\frac{n}{d}\right) 2^{d} & =2^{n}+\sum_{d \mid n, d \neq n} \mu\left(\frac{n}{d}\right) 2^{d} \\
& \geq 2^{n}-\sum_{d \mid n, d \neq n} 2^{d} \\
& \geq 2^{n}-\sum_{1 \leq d \leq\lfloor n / 2\rfloor} 2^{d} \\
& \geq 2^{n}-2^{(n / 2)+1}+2 .
\end{aligned}
$$


Consequently,

$$
1=\lim _{n \longrightarrow+\infty} \frac{2^{n}-2^{(n / 2)+1}+2}{2^{n}} \leq \lim _{n \longrightarrow+\infty} \frac{\sum_{d \mid n} \mu(n / d) 2^{d}}{2^{n}} \leq \lim _{n \longrightarrow+\infty} \frac{2^{n}+2^{(n / 2)+1}-2}{2^{n}}=1 .
$$

By the squeeze theorem,

$$
\lim _{n \longrightarrow+\infty} \frac{h(n)}{2^{n}}=\lim _{n \longrightarrow+\infty} \frac{\sum_{d \mid n} \mu(n / d) 2^{d}}{2^{n}}=1 .
$$

\section{Data Availability}

No data were used to support this study.

\section{Conflicts of Interest}

The authors declare that they have no conflicts of interest.

\section{References}

[1] A. N. Sharkovskii, "Coexistence of the cycles of a continuous mapping of the line into itself," Ukrainian Mathematical Journal, vol. 6, pp. 61-71, 1964.

[2] T.-Y. Li and J. A. Yorke, "Period three implies chaos," The American Mathematical Monthly, vol. 82, no. 10, pp. 985-992, 1975.

[3] P. Glendinning, "Topological conjugation of Lorenz maps by $\beta$-transformations," Mathematical Proceedings of the Cambridge Philosophical Society, vol. 107, no. 2, pp. 401-413, 1990.

[4] J. Milnor and W. Thurston, "On iterated maps of the interval," in Dynamical Sytems: Proceedings 1986-1987, Lecture Notes in Mathematics, Springer-Verlag, Berlin-Heidelberg-NewYork, vol. 1342, pp. 465-563, 1988.

[5] B. Susan, "The dynamics of a family of one-dimensional maps," The American Mathematical Monthly, vol. 105, no. 2, pp. 118-130, 1998.

[6] T. Lindström and H. Thunberg, "An elementary approach to dynamics and bifurcations of skew tent maps," Journal of Difference Equations and Applications, vol. 14, no. 8, pp. 819-833, 2008.

[7] K.-C. Chen and X. Dong, "On the barycenter of the tent map," Proceedings of the American Mathematical Society, vol. 138, no. 11, p. 4025, 2010.

[8] O. Pfante and J. Jost, Non-Generating Partition of Unimodular Maps, SFI Working Paper No. 2015-02-004, Santa Fe Institute, Santa Fe, NM, 2015.

[9] O. A. Ivanov, "An estimate for the number of periodical trajectories of the given period for mapping of an interval, lucas numbers, and necklaces," Vestnik St. Petersburg University: Mathematics, vol. 51, no. 4, pp. 367-372, 2018.

[10] Y. G. Shi and Y. L. Tang, "On conjugacies between asymmetric Bernoulli shifts," Journal of Mathematical Analysis and Applications, vol. 434, pp. 1792-1803, 2016.

[11] W. Parry, "Symbolic dynamics and transformations of the unit interval," Transactions of the American Mathematical Society, vol. 122, no. 2, p. 368, 1966.

[12] A. Rényi, "Representations for real numbers and their ergodic properties," Acta Mathematica Academiae Scientiarum Hungaricae, vol. 8, no. 3-4, pp. 477-493, 1957.
[13] T. M. Apostol, "Introduction to analytic number theory," in Undergraduate Texts in Mathematics, Springer-Verlag, New York-Heidelberg, 1976.

[14] E. A. Bender and J. R. Goldman, "On the applications of mobius inversion in combinatorial analysis," The American Mathematical Monthly, vol. 82, no. 8, pp. 789-803, 1975. 